\title{
Toxoplasmose na gestação
}

\author{
Bárbara B. G. R. Bártholo, ${ }^{1}$ Denise L. M. Monteiro, ${ }^{2,3^{*}}$ Alexandre J. B. Trajano, ${ }^{2,4}$ Nilson R. de Jesús ${ }^{2}$
}

\section{Resumo}

No Brasil, a prevalência de infecção aguda é de aproximadamente 1-14 casos/1.000 nascidos vivos (NV) e a de infecção congênita é de 0,2-2,0/1.000 NV. A toxoplasmose aguda durante o período gestacional é assintomática em mais de $90 \%$ dos casos. Quando sintomática, consiste em quadro gripal (febre, linfadenopatia e mal-estar) similar ao apresentado por não gestantes. A toxoplasmose congênita clássica é caracterizada pela tétrade: coriorretinite, hidrocefalia/microcefalia, calcificações cerebrais e alteração neurológica. O rastreio para T.gondii deve ser realizado com dosagem de anticorpos IgG e IgM em todas as gestantes. O diagnóstico de toxoplasmose aguda é realizado quando há IgM positivo associado a IgG negativo ou IgG e IgM positivos com baixa avidez para IgG no primeiro trimestre da gestação ou na presença de viragem sorológica. O diagnóstico de infecção fetal é feito pela identificação de T. gondii no líquido amniótico obtido por amniocentese e processado pela técnica de reação em cadeia de polimerase (PCR). O tratamento é indicado somente em casos de toxoplasmose aguda na gestação. Na suspeita de infecção aguda, deve-se iniciar imediatamente espiramicina. Se a infecção ocorrer após 30 semanas de gestação ou se houver infecção fetal comprovada, o tratamento tríplice materno com pirimetamina, sulfadiazina e ácido folínico é recomendado. O objetivo desta revisão é destacar a importância do diagnóstico precoce da toxoplasmose aguda na gestação, a fim de realizar a quimioprofilaxia da transmissão vertical, assim como o tratamento da infecção fetal na tentativa de minimizar ou evitar a manifestação fetal da toxoplasmose congênita.

Descritores: Toxoplasmose; Gravidez; Diagnóstico; Tratamento.

\section{Abstract}

\section{Toxoplasmosis in pregnancy}

Acute toxoplasmosis during pregnancy is asymptomatic in more than $90 \%$ of cases. When symptomatic, it consists of a flu-like episode (fever, lymphadenopathy and malaise) similar to the one seen in non-pregnant women. The classic congenital toxoplasmosis is characterized by the tetrad: chorioretinitis, hydrocephalus/microcephaly, cerebral calcifications and neurological abnormalities.The screening for T. gondii should be performed by dosing IgG and IgM antibodies in all pregnant women. The diagnosis of acute toxoplasmosis is made when there is positive IgM associated with negative IgG or in the presence of positive IgG and IgM with low avidity for IgG in the first trimester of pregnancy or in the presence of seroconversion. The diagnosis of fetal infection is done by detecting T. gondii in the amniotic fluid obtained by am-
1. Programa de Pós-Graduação em Ciências Médicas. Faculdade de Ciências Médicas. Universidade do Estado do Rio de Janeiro. Rio de Janeiro, RJ, Brasil.

2. Departamento de Ginecologia e Obstetrícia. Faculdade de Ciências Médicas. Universidade do Estado do Rio de Janeiro. Rio de Janeiro, RJ, Brasil.

3. Departamento de Ginecologia e Obstetrícia. Centro Universitário Serra dos Órgãos. Teresópolis, RJ, Brasil.

4. Departamento de Ginecologia e Obstetrícia. Escola de Ciências da Saúde. Universidade do Grande Rio. Rio de Janeiro, RJ, Brasil.

"Endereço para correspondência:

Núcleo Perinatal, HUPE, UERJ

Av. Prof. Manuel de Abreu, 500, 1ㅇ andar

Rio de Janeiro, RJ, Brasil. CEP: 20550-170.

E-mail: denimonteiro2@yahoo.com.br

Revista HUPE, Rio de Janeiro, 2015;14(2):65-70

doi: 10.12957/rhupe.2015.18441

Recebido em 18/09/2014. Aprovado em 13/01/2015.

niocentesis and processed by the polymerase chain reaction (PCR) technique. The treatment is indicated only in cases of acute toxoplasmosis during pregnancy. When acute infection is suspected spiramycin should be started immediately. If the infection happens after 30 weeks of pregnancy or if there is confirmed fetal infection, maternal triple therapy with pyrimethamine, sulfadiazine and folinic acid is recommended. The aim of this review is to highlight the importance of early diagnosis of acute toxoplasmosis during pregnancy in order to do the chemoprophylaxis of vertical transmission, as well as the treatment of fetal infection in an attempt to minimize or avoid fetal manifestation of congenital toxoplasmosis. In Brazil, the prevalence of acute infection is approximately1-14 cases $/ 1,000$ live births (LB) and of congenital infection is 0.2 2.0/1,000 LB.

Keywords: Toxoplasmosis; Pregnancy; Diagnosis; Treatment.

\section{Resumen}

\section{Toxoplasmosis en el embarazo}

En el Brasil, la prevalencia de la infección aguda es de aproximadamente 1-14 casos/1.000 nacidos vivos (NV) y la de infección congénita es 0,2 a 2,0/1.000 NV. La toxoplasmosis 
aguda durante el embarazo es asintomática en más del 90\% de los casos. La toxoplasmosis sintomática, consiste en un cuadro (fiebre, linfadenopatía y malestar) similar al presentado por las que no están embarazadas. La toxoplasmosis congénita clásica se caracteriza por la tétrada: coriorretinitis, hidrocefalia/microcefalia, calcificaciones cerebrales y alteraciones neurológicas. El rastreo para T. gondii debe realizarse con dosificación de anticuerpos IgG e IgM en todas las mujeres embarazadas. El diagnóstico de la toxoplasmosis aguda se realiza cuando existe IgM positivo asociado con IgG negativo o IgG e IgM positivos con baja avidez de IgG en el primer trimestre del embarazo o en presencia de viraje serológico. El diagnostico de infección fetal se realiza mediante la identificación de T. gondii en el líquido amniótico obtenido por amniocentesis y procesado

\section{Introdução}

O Toxoplasma gondii (T.gondii) é um protozoário parasita intracelular obrigatório, com ciclo de vida dependente de gatos domésticos e silvestres, únicos hospedeiros que eliminam os oocistos nas fezes. O oocisto é ingerido por outros mamíferos (por exemplo, bovinos) e liberado na luz intestinal. Humanos são infectados pela ingestão de cistos em alimentos infectados crus ou mal-cozidos ou contaminados por fezes de gatos, por transmissão vertical (TV), transfusão ou por transplante de órgãos de pessoas infectadas..$^{1-4}$

A toxoplasmose apesar de apresentar distribuição mundial, é mais prevalente em países de clima tropical, e a taxa de infecção é inversamente proporcional ao nível socioeconômico, qualidade da água e condições higiênico-sanitárias da população. Estimativas indicam que aproximadamente $25 \%$ a $30 \%$ da população humana no mundo já teve contato com o protozoário e, no Brasil, diversos estudos em pacientes gestantes evidenciam prevalência de infecção crônica de $42 \%$ a 90\%. ${ }^{5}$

A infecção fetal ocorre predominantemente em casos de infecção primária (fase aguda) durante a gestação ou em pacientes imunodeprimidas com reativação infecciosa. ${ }^{1,2} \mathrm{O}$ risco de infecção aguda com repercussões fetais é maior em mulheres sem exposição anterior à gestação, o que justifica o rastreio sorológico rotineiro, mensal ou, ao menos, trimestral dessas pacientes. ${ }^{5} \mathrm{~A}$ prevalência de infecção aguda na gestação é, aproximadamente, de 1 a 14 casos $/ 1.000$ nascidos vivos (NV). ${ }^{5}$ A TV ocorre frequentemente entre um e quatro meses após a infecção aguda, mas a placenta abriga organismos viáveis durante toda a gestação. O risco de TV aumenta com o avançar da gestação, entretanto a gravidade da doença é inversamente proporcional à idade gestacional (IG). O risco de TV da toxoplasmose por la técnica de reacción en cadena de la polimerasa (PCR). El tratamiento está indicado sólo en casos de toxoplasmosis aguda en el embarazo. En sospecha de infección aguda, se debe iniciar inmediatamente espiramicina. Si la infección se produce después de 30 semanas de embarazo o si hay infección fetal comprobada, el tratamiento triple materno con pirimetamina, sulfadiazina y ácido folínico es recomendado. El objetivo de esta revisión es poner de relieve la importancia del diagnóstico precoz de la toxoplasmosis aguda en el embarazo, con el fin de realizar la quimioprofilaxis de transmisión vertical, así como el tratamiento de la infección fetal en la tentativa de minimizar o evitar la manifestación fetal de la toxoplasmosis congénita.

Palabras clave: Toxoplasmosis; Embarazo; Diagnóstico; Tratamiento.

aguda durante o primeiro trimestre é de $6 \%$ e no terceiro trimestre é de $60 \%$ a $81 \%$. A taxa de infecção fetal quando não tratada durante a gestação é de $20 \%$ a 50\% e a prevalência de infecção congênita é de aproximadamente 0,2 a 2/1.000 NV. 5,6

\section{Aspectos clínicos}

As apresentações clínicas da infecção são resultado do dano direto ao organismo e à resposta imunológica subsequente à parasitemia e morte celular. Também há evidências que as consequências gestacionais estão associadas ao aumento da resposta inflamatória decidual levando à apoptose de células e à necrose na placenta.? Na maioria das gestantes (>90\%), a infecção é assintomática e tem resolução espontânea. A manifestação clínica mais frequente ésemelhante a um quadro gripal, com febre, linfadenopatia e mal-estar semelhante às não gestantes. O período de incubação é de cinco a 18 dias após a exposição. ${ }^{1}$

A toxoplasmose congênita clássica é caracterizada pela tétrade descrita por Sabin em 1942: coriorretinite, hidrocefalia ou microcefalia, calcificações cerebrais e alteração neurológica. Outras complicações características da toxoplasmose congênita são: abortamento, restrição de crescimento fetal,1 hidropsia, parto pré-termo, morte neonatal, alterações hematológicas e deficit de desenvolvimento neurocognitivo., ${ }^{2,3}$

\section{Diagnóstico}

O screening para T. gondii deve ser realizado com dosagem de anticorpos imunoglobulina G (IgG) e (Imunoglobulina $\mathrm{G}$ ) e imunoglobulina M (IgM) para toxoplasmose em todas as gestantes, idealmente durante o primeiro trimestre de gestação e, em mulheres soronegativas, mensal ou trimestralmente. ${ }^{2}$ 
O diagnóstico de toxoplasmose aguda na gestante é realizado pela presença de: IgM para toxoplasmose positivo associado a IgG negativo (ou aumento de quatro vezes ou mais na IgG), devendo ser iniciada espiramicina e solicitada nova sorologia em três meses (na permanência de IgG negativo, suspende-se a medicação e realiza-se sorologias seriadas de $3 / 3$ meses). A infecção subaguda ou recente ocorre quando IgG e IgM são positivas com teste de avidez para IgG baixo no primeiro trimestre. ${ }^{1,8,9}$ A figura 1 é representativa do diagnóstico sorológico e conduta da toxoplasmose na gestação.

A IgM aparece aproximadamente na primeira ou segunda semana após a infecção, com o pico ocorrendo em seis a oito semanas, quando declina a partir daí. No entanto, pode permanecer positiva até quatro a seis meses após o início da infecção ou persistir em títulos baixos por mais 12 meses (IgM residual). Devido à elevada incidência de testes falso-positivos, o exame deve ser repetido em nova amostra com intervalo de duas semanas e, se possível, em laboratórios de referência. Somente quando identificamos a soroconversão, podemos confirmar a infecção aguda apenas com a presença de $\operatorname{IgM} .^{10-12}$

A IgG aparece mais tardiamente, cerca de duas a quatro semanas após a infecção, apresenta pico com 12 semanas a seis meses após a infecção aguda, permanecendo positiva por toda a vida. ${ }^{1}$ Nos casos de IgG e IgM positivas, utiliza-se o teste de avidez da IgG para definir a necessidade do tratamento. Quando a avidezé baixa (<30\%), a infecção é recente (tendo ocorrido em menos de quatro meses) e merece tratamento. Quando a avidezé alta (>60\%), dispensa tratamento por se tratar de infecção mais antiga, sem risco de acometimento fetal. O teste é muito mais útil quando a avidez é alta, porque alguns fatores podem retardar a maturação da IgG, como o uso da espiramicina, por exemplo. A pesquisa de anticorpos IgA e IgE pode fornecer mais acurácia no diagnóstico de infecção materna. ${ }^{5,10-12}$

Ocasionalmente, o diagnóstico de infecção em mulheres gestantes é primariamente considerado quando há achados ultrassonográficos que revelam a presença de anormalidades fetais. ${ }^{2}$

Pacientes imunocompetentes com IgG positivo e IgM negativo para toxoplasmose são consideradas com imunidade remota e, consequentemente, infecção crônica, dispensando sorologias posteriores ou cuidados específicos. $^{10,11}$

\section{Diagnóstico de infecção fetal}

As pacientes com suspeição de toxoplasmose aguda diagnosticadas durante a gestação devem ser submetidas à amniocentese em até quatro semanas do diagnóstico e após 18 semanas de gestação para identificar T. gondii no líquido amniótico através da técnica de PCR (sensibilidade de $81 \%$ a $90 \%$ e especificidade de $96 \%$ a 100\%. Não há resultado falso-positivo e o valor preditivo positivo é de $100 \%)^{2}$ A presença desse protozoário no líquido amniótico indica infecção do concepto. ${ }^{3}$

Outras possíveis indicações de amniocentese com realização de PCR são: sorologia que não confirma ou exclui a infecção aguda materna; presença de achados ultrassonográficos fetais anormais, como calcificação

Figura 1. Diagnóstico e conduta da toxoplasmose na gestação.

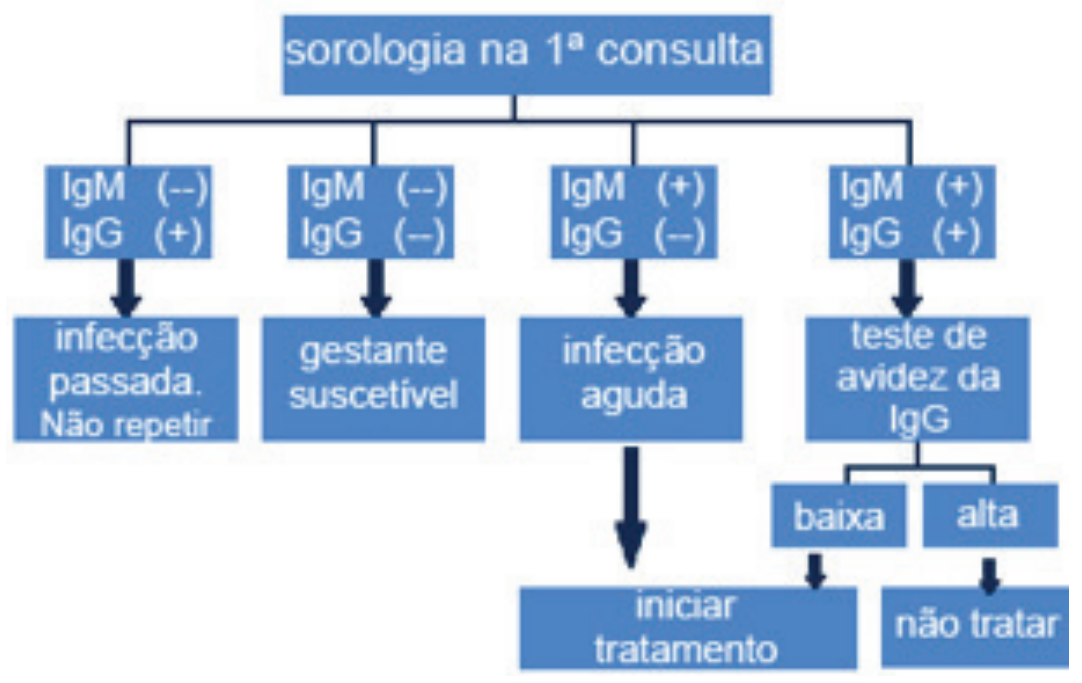




\section{Artigo de revisão}

intracraniana, microcefalia, hidrocefalia, ascite, hepatoesplenomegalia ou restrição do crescimento intrauterino severo; e mulheres imunossuprimidas com risco de reativação da infecção latente, exceto aquelas com vírus da imunodeficiência humana (HIV).1,3

Os riscos inerentes à amniocentese são: isoimunização materna em gestantes com fator Rh negativo, devendo ser submetidas à aplicação da imunoglobulina anti-D antes do procedimento; abortamento (1\%) e infecção. ${ }^{13-16}$ O risco de sepse grave materna é provavelmente menor que $1 / 1.000$ procedimentos. A infecção pode ser causada pela punção inadvertida de intestino, contaminação da pele ou organismos presentes no gel ou probe do ultrassom..$^{15}$ Ressalta-se, ainda, o risco de transmissão vertical de doenças virais, como hepatites B e C e HIV, durante o procedimento., ${ }^{3,1}$ Traumas maternofetais são descritos em publicações de casos, porém são raros e, provavelmente, relacionados ao inadequado controle ultrassonográfico do exame.16

\section{Prevenção primária}

Pacientes com sorologias IgG e IgM negativos são suscetíveis à infecção, devendo ser informadas sobre os mecanismos de prevenção primária da infecção, incluindo higiene adequada, manipulação e ingestão dos alimentos; evitar ingestão de carnes cruas, malcozidas, ou mal-passadas; evitar contato com solo e terra de jardins sem proteção individual (botas e luvas).6,11

\section{Prevenção da transmissão vertical e tratamento pré-natal}

Até o momento não há estudos controlados randomizados capazes de confirmar que o tratamento materno é capaz de reduzir o risco da transmissão vertical. Os resultados mais consistentes foram relatados pela revisão sistemática realizada pelo SYROCOT StudyGroup, 2007. ${ }^{17}$ Os autores não encontraram evidências de que o tratamento pré-natal seja capaz de reduzir as lesões intracranianas, as retinocondrites ou a transmissão vertical, entretanto encontraram evidências de que pode haver redução nas sequelas neurológicas decorrentes da infecção congênita. ${ }^{17}$ Apesar da falta de evidência sobre eficácia do tratamento, há consenso de que ele deve ser oferecido à paciente com o diagnóstico de infecção aguda (IgM positiva e IgG negativa) e recente (IgM e IgG positivas com baixa avidez de $\operatorname{IgG})^{1,3}$ Os regimes terapêuticos variam de acordo com a presença ou não de infecção fetal e os utilizados no Núcleo Perinatal são os seguintes: a) PCR do líquido amniótico negativo - utilizar espiramicina $1 \mathrm{~g}$ (3.000.000 UI ou 3,0 MUI) VO de oito em oito horas até o final da gestação.11 A espiramicina parece não tratar o concepto, pois não atravessa a barreira placentária, mas previne a transmissão placentária em até 60\% das vezes. Os efeitos colaterais mais comumente observados são: sintomas gastrintestinais, diarreia, tonteira e anorexia;

b) PCR do líquido amniótico positivo - utilizar espiramicina conforme descrito anteriormente, até o diagnóstico de infecção fetal. Após o diagnóstico, substituir a espiramicina pela combinação de sulfadiazina $1 \mathrm{~g}$ VO de seis em seis horas e pirimetamina $25 \mathrm{mg}$ VO uma vez ao dia. Esse esquema deve ser acompanhado do ácido folínico $15 \mathrm{mg}$ VO em dias alternados, a fim de prevenir as alterações hematológicas induzidas pela pirimetamina.18 $\mathrm{Na} 36^{\mathrm{a}}$ semana, esse esquema deve ser suspenso e a paciente retornará ao uso da espiramicina.

Os efeitos colaterais mais frequentes relacionados ao uso de sulfadiazina são: alergia, rash, anemia, intolerância gastrintestinal e hepatotoxicidade. O uso de pirimetamina está associado à anemia, leucopenia, plaquetopenia, teratogênese e intolerância gastrintestinal. As pacientes devem realizar hemograma e contagem de plaquetas a cada dez dias enquanto estiverem utilizando estas medicações. ${ }^{10,11}$

Os esquemas utilizados no tratamento da toxoplasmose aguda na gestação são variáveis. Na França, o esquema terapêutico prescrito é semelhante ao preconizado pelo Ministério da Saúde (MS). Ao contrário, na Áustria, mulheres são inicialmente tratadas com pirimetamina e sulfadiazina após 15 semanas de gestação e o esquema é modificado para espiramicina após diagnóstico fetal negativo. ${ }^{6}$

No protocolo do MS do Brasil, o tratamento tríplice inclui: pirimetamina $25 \mathrm{mg}$ de $12 \mathrm{em} 12$ horas por via oral, sulfadiazina $1.500 \mathrm{mg}$ de $12 \mathrm{em} 12$ horas por via oral e ácido folínico $10 \mathrm{mg}$ por dia.11

Vale ressaltar que há questionamento sobre a eficácia do tratamento da toxoplasmose aguda na gestação. Estudo realizado com 1.438 mulheres tratadas após diagnóstico no rastreio pré-natal revelou fraca associação entre o tratamento iniciado dentro de três semanas da soroconversão e a TV. ${ }^{6}$ Em outro estudo prospectivo, observou-se 255 infantes infectados e concluiu-se que nenhum tratamento pré-natal dentro das primeiras quatro semanas de soroconversão foi associado à redução do risco de lesões intracranianas. ${ }^{4}$ Outro recente es- 
tudo europeu incluiu 293 fetos infectados (189 tratados, 284 nascidos vivos) de 14 centros com média de quatro anos de seguimento e revelou que nenhum tratamento foi associado com redução do risco de sequelas neurológicas graves ou morte. Neste mesmo estudo não houve diferença significativa na resposta ao tratamento entre espiramicina e pirimetamina. ${ }^{4}$

É importante ratificar a necessidade de notificação dos casos de toxoplasmose aguda na gestação à Vigilância Epidemiológica. ${ }^{11}$

\section{Acompanhamento das gestantes}

Na suspeição ou confirmação de infecção aguda por T. gondii durante a gestação, deve-se realizar ultrassonografia (USG) obstétrica mensalmente para avaliação fetal. ${ }^{2}$ A USG pode revelar a presença de anormalidades fetais, como hidrocefalia, calcificação hepática e cerebral, esplenomegalia e ascite. ${ }^{1}$

As pacientes em esquema tríplice de tratamento devem ser submetidas à avaliação frequente com hemograma, visto que a pirimetamina e sulfadiazina provocam pancitopenia. ${ }^{2}$

\section{Gestantes imunocomprometidas}

A imunidade do hospedeiro para T. gondii é mediada primariamente pelos linfócitos T, logo pacientes com infecção pelo HIV e aquelas submetidas à terapia imunossupressora cronicamente são particularmente suscetíveis à reativação da infecção. Nessas pacientes, a disfunção do sistema nervoso central é a mais comum manifestação da infecção. Outros achados típicos incluem encefalite, meningoencefalite, lesões com efeito de massa intracerebral, pneumonite, miocardite e linfadenopatia generalizada., ${ }^{1,3}$ As gestantes com imunossupressão e infecção crônica por T.gondii podem transmitir o parasita ao feto (em $4 \%$ dos casos), especialmente quando apresentam contagem de CD4 abaixo de 100 cels/mm., ${ }^{3,2,19} \mathrm{O}$ risco de TV é baixo quando a contagem de CD4 é maior que 200 cels/mm.,18,20

Dessa forma, mulheres infectadas cronicamente pelo T. gondii com HIV devem ser tratadas de acordo com a contagem de CD4:

a) CD4 < 200 cels $/ \mathrm{mm}^{3}$ - sulfametoxazol-trimetoprim (trimetoprim $80 \mathrm{mg}$ e sulfametoxazol 400 mg uma vez ao dia) para prevenir a reativação e a transmissão do parasita ao feto. $\mathrm{O}$ trimetoprim é frequentemente evitado no primeiro trimestre por ser antagonista do ácido fólico;

b) CD4 > 200 cels $/ \mathrm{mm}^{3}$ ou imunocomprometidas não infectadas pelo HIV - espiramicina durante todo o período gestacional;

c) deve-se também proceder a realização de USG fetal mensal para pacientes gestantes imunocomprometidas infectadas cronicamente pelo T. gondii. ${ }^{2}$

\section{Conclusão}

A sorologia para T.gondii deve ser solicitada na primeira consulta de pré-natal e de três em três meses. Na suspeição ou certeza de diagnóstico de toxoplasmose aguda na gestação, devem-se realizar PCR do LA, USG mensal e quimioprofilaxia/tratamento medicamentoso da infecção, a fim de reduzir a TV da infecção e a gravidade da toxoplasmose congênita.

\section{Referências}

1. Paquet $\mathrm{C}$, Trois-Rivières $\mathrm{QC}$, Yudin $\mathrm{MH}$, Toronto $\mathrm{ON}$ and Infectious Disease Committee. Toxoplasmosis in Pregnancy: prevention, screening, and treatment. J Obstet Gynecol Can 2013; 35(1 e Suppl A):S1-S7.

2. Montoya JG, Remington JS. Management of Toxoplasma gondii infection during pregnancy. Clin Infect Dis. 2008; 47(4):554-66. doi: 10.1086/590149.

3. Duff P. Maternal and perinatal infection bacterial. In: Gabbe SG; Niebyl JR; Simpson JL; et al. Obstetrics normal and problem a pregnancies. Philadelphia 2012; 6 ed.; p.1140-55.

4. Gangneux FR. It is not only the cat that did it: How to prevent and treat congenital toxoplasmosis. Journal of Infection. 2014; 68: S125 - S133. http://dx.doi. org/10.1016/j.jinf.2013.09.023.

5. Pessanha TM, Carvalho M, Pone MV, et al Diagnostic and therapeutic management of toxoplasmosis in pregnancy and the effect in the newborn. Rev. Paul. Pediatr 2011; 29(3): 341-7.

6. Thiébaut R. Effectiveness of prenatal treatment for congenital toxoplasmosis: a meta-analysis of individual patients' data. Lancet 2007; 369(9556): 115-22.

7. Coutinho LB, Gomes AO, Araújo ECB, et al. The impaired pregnancy outcome in murine congenital toxoplasmosis is associated with a pro-inflammatory immune response, but not correlated with decidual inducible nitric oxide synthase expression. Int J Parasitol. 2012; 42(4): 341-52. doi: 10.1016/j.ijpara.2012.01.006.

8. Reis MM, Tessaro MM, D, Azevedo PA. Toxoplasma-IgM and IgG-avidity in single samplesfromareaswith a highinfectionrate can determine therisk of mother-to-childtransmission. RevInstMedTrop Sao Paulo. 2006; 48(2): 93-8.

9. Lappalainen M, Koskiniemi M, Hilesmaa V, et al Outcome of children after maternal primary Toxoplasma infection during pregnancy with emphasis on avidity of specific IgG. Pediatr Infect Dis J. 1995; 14(5):354-61.

10. Andrade JQ, Amorim AGF, Zugaib M. Toxoplasmose. In: Montenegro, Rezende. Obstetrícia. Rio de Janeiro. 2010;(11):751-7.

11. Ministério da Saúde, Brasil. Gestação de alto risco - manual técnico. 2012; (5): 115-8. 


\section{Artigo de revisão}

12. Zaconeta ACM, Cabral ACV, Filho BJA, et al. Toxoplasmose na gestação. FEBRASGO. 2011;143-7.

13. Meleti D, De Oliveira LG, Araujo Júnior E, et al. Evaluation of passage of fetal erythrocytes into maternal circulation after invasive obstetric procedures. J Obstet Gynecol Res. 2013; 39(9):1374-82.doi: 10.1111/jog.12073.

14. Tabor A, Vestergaard CH, Lidegaard F. Fetal loss rate after chorionic villus sampling and amniocentesis: an 11-year national registry study.Ultrasound Obstet Gynecol. 2009; 34(1):19-24.doi: 10.1002/uog.6377.

15. RCOG Green-top Guideline No. 8 (Revised 2013). Amniocentesis and Chorionic Villus Sampling.

16. HKCOG Guideline N 4 (Revised 2009). Guidelines for Amniocentesis and Chorionic Villus Sampling (CVS).
17. Syrocot (Systematic review on congenital toxoplasmosis) study group. Thiébaut R, Leproust S, Chêne G, et al. Effectiveness of prenatal treatment for congenital toxoplasmosis: a meta-analysis of individual patients' data. Lancet. 2007; 369(9556):115-22.

18. Binquet $\mathrm{C}$, Wallon $\mathrm{M}$, Metral $\mathrm{P}$, et al. Toxoplasmosis seroconversion in pregnant women. The differing attitudes in France. Presse Med. 2004; 33(12 Pt 1):775-9.

19. Bollani L, Strocchio L, Stronati M. Congenital toxoplasmosis. Early Hum Dev. 2013; 89 S4:S70-S71.

20. Feldman DM, Timmis D, Borgida AF. Toxoplasmosis, parvovirus, and cytomegalovirus in pregnancy. Clin Lab Med. 2010; 30(3):709-720.doi: 10.1016/j.cll.2010.04.009. 